\title{
Association of HLA antigens with the clinical course of sarcoidosis and familial disease
}

\author{
Halil Yanardag', Cuneyt Tetikkurt2', Muammer Bilir1, Erkan Yılmaz³ \\ 1 Department of Internal Medicine Medicine, Cerrahpasa Medical Faculty, Istanbul University \\ 2 Department of Pulmonary Medicine, Cerrahpasa Medical Faculty, Istanbul University \\ 3 Tissue Typing Laboratory, Cerrahpasa Medical Faculty, Istanbul University, Turkey
}

\begin{abstract}
Patients with sarcoidosis usually have a benign course and a favourable prognosis. Although spontaneous remission is common, a progressive disease with a severe prognosis occurs in a small but significant number of patients. The aim of this study was to evaluate the potential significance of HLA antigens as a clinical marker on the outcome of sarcoidosis patients. We conducted a retrospective cohort study for HLA class I and II allels in 74 sarcoidosis patients and 72 healthy transplant donors. Bronchoscopy and bronchial biopsies were performed in each patient. Two or more positive bronchial biopsy samples revealing noncaseified granulomatous inflammation was defined as diffuse while one positive biopsy sample was designated as limited endobronchial disease. Three or more extrapulmonary organ involvement was denoted as extensive and involvement of two or less organs was designated as limited extrapulmonary organ disease. The patients were followed-up at least for eight years. Incidence of progressive disease was significantly high in patients with positive HLA-DRB1*07, DRB1*14 ( $<<0.05)$ and DRB1*15 ( $<<0.001)$ allels. HLA-DRB1*14 and DRB1*15 were associated with extensive extrapulmonary organ disease $(\mathrm{p}<0.001)$. HLA-DRB1 ${ }^{*} 14(\mathrm{p}<0.05)$ and DRB1*15
\end{abstract}

Corresponding author: Prof. Cuneyt Tetikkurt, Tanzimat Sok. Serkan Apt. No:8/16, 34728 Caddebostan, Istanbul, Turkey.

Tel. +90.216.36019 77 - Fax +90.212.5870217. E mail: tetikkurt@gmail.com

Key words: Sarcoidosis; HLA; prognosis; HLA-DRB; human leukocyte antigen.

Contributions: HY, study design, patient and data evaluation; CT, contribution to manuscript preparation; $\mathrm{MB}$, contribution to patient follow-up and statistical analysis; EY, contribution to HLA tissue typing and analysis of HLA data.

Conflict of interest: the authors declare no conflict of interest.

Received for publication: 4 May 2017

Accepted for publication: 26 August 2017

CC Copyright H. Yanardag et al., 2017

Tipografia PI-ME Editrice, Italy

Monaldi Archives for Chest Disease 2017; 87:835

doi: 10.4081/monaldi.2017.835

This article is distributed under the terms of the Creative Commons Attribution Noncommercial License (by-nc 4.0) which permits any noncommercial use, distribution, and reproduction in any medium, provided the original author(s) and source are credited. $(\mathrm{p}<0.001)$ were significantly more frequent in patients with diffuse endobronchial involvement. Incidence of familial disease was $14.8 \%$ with a $6.7 \%$ identical HLA typing. Presence of HLA class I and II allels may influence the severity and prognosis of sarcoidosis significantly. Apart from defining genetic susceptibility, HLA class I and class II allels appear to be relevant and crucial markers for the clinical outcome of sarcoidosis. Distinct heterogenity of sarcoidosis may arise from the particular presence of different allels in invidual patients.

\section{Introduction}

The clinical expression, natural history, and prognosis of sarcoidosis are highly variable and its course is often unpredictable. Presentation, clinical manifestations, and outcome vary with the organs involved [1-3]. The cause of sarcoidosis and its wide phenotopic differences are not yet fully understood [4]. Prevalence in different ethnic groups, familial occurrence, and its high incidence among monozygotic twins suggests genetic predisposition and several studies indicate that HLA antigens are more frequent in patients with sarcoidosis and genetic factors may play an important role in modifying the risk for the disease, its phenotype, and outcome [1,5-10].

In many patients with sarcoidosis the disease resolves spontaneously. Even if the disease is persistent it may not require treatment. However, a substantial minority of sarcoidosis patients has a severe, chronic, or progressive disease with concomitant morbidity and mortality. Heterogeneity of sarcoidosis may arise from the interaction of environmental, humoral, and genetic factors that result in different or variable disease patterns or clinical manifestations that pose difficulties in both diagnosis and treatment of these patients [2-4].

Until now, no study has comprehensively established which features determine the chance of spontaneous resolution or serious organ involvement [4]. The strong correlation between HLA allels and the genetic profile indicate that HLA typing may be a useful marker for the evaluation of sarcoidosis patients in regard to their variable clinical profile and prognosis. The aim of this study is to investigate the correlation between HLA allels and the clinical outcome in Caucasian patients with sarcoidosis in regard to disease severity, organ involvement, and prognosis.

\section{Materials and Methods}

Seventy-four biopsy proven Caucasian sarcoidosis patients attending Cerrahpasa Medical Faculty Internal Medicine department between March 1990 and April 2017 were evaluated for HLA I and HLA II allels and 72 renal transplant donors were included as a control group. The study follows a retrospective cohort design and has been approved 
by the IRB/Ethics Committee of Cerrahpasa Medical Faculty (02/82533). Each patient had provided informed and written consent. Patients fulfilled the American Thoracic Society/European Respiratory Society criteria of sarcoidosis [1]. All subjects underwent blood biochemistry, urine analysis, pulmonary function tests, $\operatorname{DLCO}_{\mathrm{A}}$, chest $\mathrm{x}$ ray, thorax CT, and FOB. Microlymphocytotoxicity method was used for HLA analysis.

Chest roentgenograms were staged according to the DeRemee classification [11]. The pulmonary function tests and $\mathrm{DLCO} / \mathrm{N}_{\mathrm{A}}$ were interpreted in accordance with the guidelines of ATS [12]. For skin and ocular sarcoidosis involvement all patients were screened by a dermatologist and an ophthalmologist. Central nervous system involvement was considered to exist if neurologic findings were positive, a lesion was confirmed by CT or MRI, and identified by a consultant neurologist. Sputum, bronchial lavage or BAL culture was done to exclude infection. FOB was performed under local anesthesia in all patients. Abnormal airways were defined as having mucosal thickening, erythematous, miliary or nodular lesions. In patients with normal appearing mucosa eight biopsies were taken from the main, secondary carenas and bronchial mucosa. In patients with abnormal mucosa six to eight biopsies were taken from the lesion sites. The patients were classified into three groups according to the biopsy results: i) no endobronchial involvement (NEI); ii) limited endobronchial involvement (LEI): one biopsy site positive; and iii) diffuse endobronchial involvement (DEI): two or more biopsy sites positive for noncaseified granulomas. Organ involvement was classified into two groups as limited (less than three organs involved) or extensive (three or more organs) extrapulmonary organ disease.

Progressive disease was defined as advancing stage, increase in radiologic stage, deterioration of pulmonary function or CT findings, persistent systemic or organ symptoms, presence of hypercalcemia or hypercalcuria, and three or more organ involvement. The patients who had manifestations of persistent disease after five years were classified as having a chronic nonresolving sarcoidosis. Patients without signs of active disease were denoted as stable disease [13-15]. Thirty patients received corticosteroids, four patients received azathioprine, and two patients received methotrexate for treatment. Clinical findings were first evaluated one month after initial diagnosis and every 3 months thereafter. The mean follow-up period was eight years for each patient.

Associations between HLA markers and sarcoidosis in regard to progressive disease, organ, and endobronchial involvement were analysed by contingency tables. $X^{2}$ with Yates' correction and Fishers' probability values were calculated for HLA antigens. Odds ratios with $95 \%$ confidence intervals were utilized to evaluate the strength of associations. Logistic regression was applied to determine the effect of age, gender and endobronchial involvement on prognosis. Kruskal-Wallis test and Bonferronni corrected two way Mann-Whitney test were used for com- parison of the groups. Probability values were corrected for multiple comparisons. All tests were two tailed and a p value less than 0.05 was used as the threshold for statistical significance. Analyses were done using software (ver. SPSS 22.0).

\section{Results}

HLA typing for class I and II antigens were investigated in 74 sarcoidosis patients and 72 healthy controls. Forty two of these patients were women and the mean age was $38.2 \pm 9.2$, ranging from 26 to 42 years. Demographic data are presented in Table 1. The frequencies of HLA alleles in healthy subjects and sarcoidosis patients are shown in Table 2. The disease course was persistent in 34 patients $(34 / 74,48.6 \%)$ while the disease was stable in 40 sarcoidosis patients (40/74, 51.4\%). There were significant alterations in the frequencies of HLA antigens in the sarcoidosis patients. HLA-B*27 (8\%) and HLA-B*DR7 (6\%) antigens were less frequent $(\mathrm{p}<0.01)$ in sarcoidosis patients than the control subjects (32.4\% and 38.1\%). Logistic regression with Kruskal-Wallis test and Bonferronni corrected two way Mann-Whitney test revealed no significant difference of age and gender on prognosis, endobronchial, or extra-pulmonary organ involvement, and HLA alleles. Spontaneous resolution was more frequent in HLA-DRB1 ${ }^{*} 01$ and HLA-DRB1*03 positive patients. HLA-DRB $1 * 01$ and HLA-DRB ${ }^{*} 03$ alleles were overpresented in the resolving disease group of patients $(68.2 \%$ and $62.7 \%)$ compared with the healthy control subjects (18.6\% and $16.8 \%$, $\mathrm{p}<0.001)$ and these patients recovered in 24 months. HLA-DRB $1 * 017$ (71.4\%) patients had the best initial FVC and DLC0 values $(\mathrm{p}<0.001)$. In HLA-DRB1*015 (61.3\%) positive patients, FVC and DLC0 values decreased significantly $(\mathrm{p}<0.01)$ during the follow-up period (Table 3 ).

Table 1. Demographic data and laboratory findings.

\begin{tabular}{ll}
\hline Demographics & \\
Age: $38.2 \pm 9.2$ & \\
Female: 42 & \\
Spirometry & $74.8 \pm 14.6$ \\
FEVl, \% predicted & $80.2 \pm 12.4$ \\
FVC, \% predicted & $86.2 \pm 16.2$ \\
TLC, \% predicted & $82.6 \pm 8.8$ \\
DLCONA, \% predicted & \\
Laboratory & $8.92 \pm 0.96$ \\
Serum Ca, mg/dL & $259.6 \pm 34.8$ \\
Urinary Ca, mg/day & $9.8 \pm 14.4$ \\
Serum ACE, IU/L
\end{tabular}

Table 2. Distribution of HLA alleles among sarcoidosis patients and the control subjects.

\begin{tabular}{|c|c|c|c|c|}
\hline HLA allele & $\begin{array}{c}\text { Total patients } \\
\mathrm{n}=74(\%)\end{array}$ & $\begin{array}{c}\text { Stable disease } \\
n=40(\%)\end{array}$ & $\begin{array}{c}\text { Chronic disease } \\
n=34(\%)\end{array}$ & $\begin{array}{c}\text { Control subjects } \\
n=72(\%)\end{array}$ \\
\hline HLA-DRB1*01 & $51(68.9)$ & $25(62.5)$ & $15(20.2)$ & $14(19.4)$ \\
\hline HLA-DRB1 ${ }^{*} 03$ & $48(64.8)$ & $27(70.6)$ & $17(22.9)$ & $13(18.1)$ \\
\hline HLA-DRB1*07 & $49(66.2)$ & $8(20.0)$ & $40(54.1)$ & $17(23.6)$ \\
\hline HLA-DRB1*14 & $47(63.5)$ & $7(17.5)$ & $36(48.6)$ & $16(22.2)$ \\
\hline HLA-DRB1*15 & $53(71.6)$ & $9(22.5)$ & $43(58.1)$ & $15(20.8)$ \\
\hline HLA-DRB1*17 & $56(75.6)$ & $27(70.0)$ & $18(24.3)$ & $12(16.6)$ \\
\hline HLA-B*03 & $54(72.9)$ & $29(72.5)$ & $14(18.9)$ & $11(15.2)$ \\
\hline HLA-B*07 & $55(74.3)$ & $26(65.0)$ & $16(21.6)$ & $17(23.6)$ \\
\hline HLA-B*08 & $52(70.2)$ & $30(75.0)$ & $15(20.2)$ & $10(13.8)$ \\
\hline
\end{tabular}


Initial chest X-ray images revealed more advanced radiologic disease stages of II or III in most of the HLA-DRB1*03 (63.7\%) and HLA-DRB1*07 $(61.6 \%)$ positive patients $(\mathrm{p}<0.01)$ while most patients with HLA-DRB1*17 (72.1\%) positive patients presented with stage I sarcoidosis $(\mathrm{p}<0.01)$. HLA-DRB $1 * 07$, HLA-DRB1 ${ }^{*} 14$, and HLA-DRB $1 * 15$ were strongly associated with progressive chronic pulmonary disease compared with the control subjects (51.2\% vs $26.4 \%, 48.6$ vs 24.7, and 54.7 vs $28.3 \%$ ) (Table 3 , Figure 1). There was no statistically significant difference for $\mathrm{A1}, \mathrm{A} 2, \mathrm{~A} 3, \mathrm{A10}, \mathrm{A11}$, A19, A26, A28-32, A36, B8, B12-18, B21, B22, B35, B37, B41, B44, B48, B51, $\mathrm{B} 55, \mathrm{~B} 60, \mathrm{~B} 62, \mathrm{~B} 67, \mathrm{DRB} 1 * 06, \mathrm{DRB} 1{ }^{*} 08-{ }^{*} 13$, and DRB1*16-*18 antigens between sarcoidosis patients and the control group.
HLA-DRB ${ }^{*} 14$ and HLA-DRB ${ }^{*} 15$ positivity was more frequent $(\mathrm{p}<0.05, \mathrm{p}<0.01)$ in patients with severe organ involvement (Table 3 , Figure 2) compared with the healthy control subjects $(68.2 \%$ and $59.8 \%$ vs $24.7 \%$ and $28.3 \%$ ). Diffuse endobronchial disease was more common HLA-DRB1*14 (54.3\%) and HLA-DRB1*15 (56.8\%) positive patients than the control group $(19.7 \%$ and $21.3 \%$; $<<0.05, \mathrm{p}<0.01)$ (Table 3, Figure 3). Incidence of stable disease was highest among patients with HLA-B*03 (72.3\%), HLA-B*07 (69.2\%), and HLA-B*08 (67.4\%) positivity in regard to the control group $(21.1 \%, 24.3 \%$, and $28.4 \%$; $<<0.001$ ). Two patients with HLA-DRB1*07 positivity died of respiratory insufficiency due to sarcoidosis. One of these patients

Table 3. HLA-DRB1 allels and sarcoidosis.

\begin{tabular}{|c|c|c|c|c|}
\hline HLA allel & Spontaneous resolution & p value & OR & CI $(95 \%)$ \\
\hline $\mathrm{DRB} 1 * 07$ & $26 / 74$ & $<0.01$ & 4.8 & $(2.4-14.1)$ \\
\hline DRB1*03 & $24 / 74$ & $<0.05$ & 3.6 & $(1.4-8.2)$ \\
\hline \multicolumn{5}{|c|}{ Best initial $\mathrm{FEV}_{1}, \mathrm{FVC}$ and $\mathrm{DLCO}$} \\
\hline DRB1*17 & $32 / 74$ & $<0.01$ & 3.2 & $(2.6-9.4)$ \\
\hline \multicolumn{5}{|c|}{ Advanced disease in x-ray } \\
\hline DRB1*03 & $22 / 74$ & $<0.05$ & 3.8 & $(1.5-16.8)$ \\
\hline DRB1*07 & $24 / 74$ & $<0.01$ & 4.2 & $(2.4-9.6)$ \\
\hline \multicolumn{5}{|c|}{ Deterioration of PFT } \\
\hline DRB1*15 & $25 / 74$ & $<0.05$ & 4.7 & $(1.8-14.2)$ \\
\hline \multicolumn{5}{|c|}{ Extensive organ involvement } \\
\hline DRB1*14 & $26 / 74$ & $<0.05$ & 5.2 & $(1.9-8.4)$ \\
\hline DRB1*15 & $28 / 74$ & $<0.01$ & 4.9 & $2.6-9.8)$ \\
\hline \multicolumn{5}{|c|}{ Diffuse endobronchial disease } \\
\hline DRB1*14 & $24 / 74$ & $<0.05$ & 6.4 & $(2.1-14.8)$ \\
\hline DRB1*15 & $27 / 74$ & $<0.01$ & 5.8 & $(2.8-11.2)$ \\
\hline \multicolumn{5}{|c|}{ Chronic and progressive sarcoidosis } \\
\hline DRB1*07 & $29 / 74$ & $<0.05$ & 5.4 & $(1.8-16.2)$ \\
\hline DRB1*14 & $31 / 74$ & $<0.05$ & 5.2 & (2.4-17.6) \\
\hline DRB1*15 & $34 / 74$ & $<0.01$ & 4.6 & $(1.6-14.4)$ \\
\hline
\end{tabular}

$\chi^{2}$ test, Kruskal-Wallis test and Bonferronni corrected two way Mann-Whitney test were used for statistical analysis.

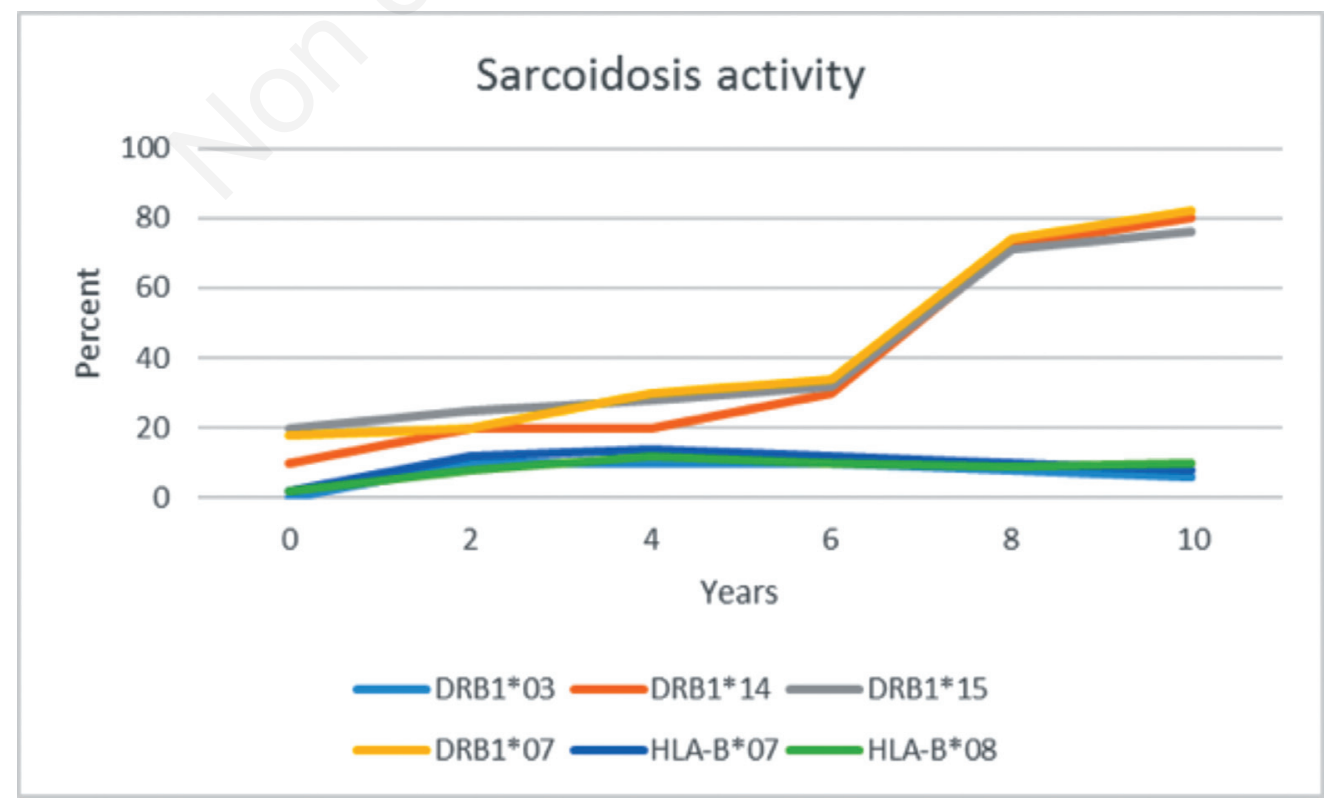

Figure 1. Sarcoidosis activity and HLA association. 
had two cerebral granulomas. Seventy-six per cent of the DRB1*17 positive patients showed spontaneous resolution $(\mathrm{p}<0.01)$ compared to control group (29.7\%). Specific organ involvement was evenly distributed between HLA allels without any significant statistical difference. Among all these sarcoidosis patients three sisters, two sisters, two sisters, two brothers, one sister and one brother had identical HLA typing. The distribution of HLA alleles among siblings is shown in Table 4. The incidence of familial disease was $17.6 \%$ and identical HLA typing was $7.4 \%$ in our patients.

Table 4. Identical HLA alleles among sibling sarcoidosis patients.

\section{Siblings}

Two sisters

Two sisters

Three sisters

Two brothers

Two brothers

\section{Identical HLA alleles}

A25, A68, B35, B4, DR11, DR13, DQ2, DQ6

A2, A3, B15, B38, DR11, DR13

A11, A25, B6, B22, DQ4, DQ7, DR14, DR51, DR52

A2, B46, B51, DR11, DR13

A1, A3, B35, B57, DR13, DR16

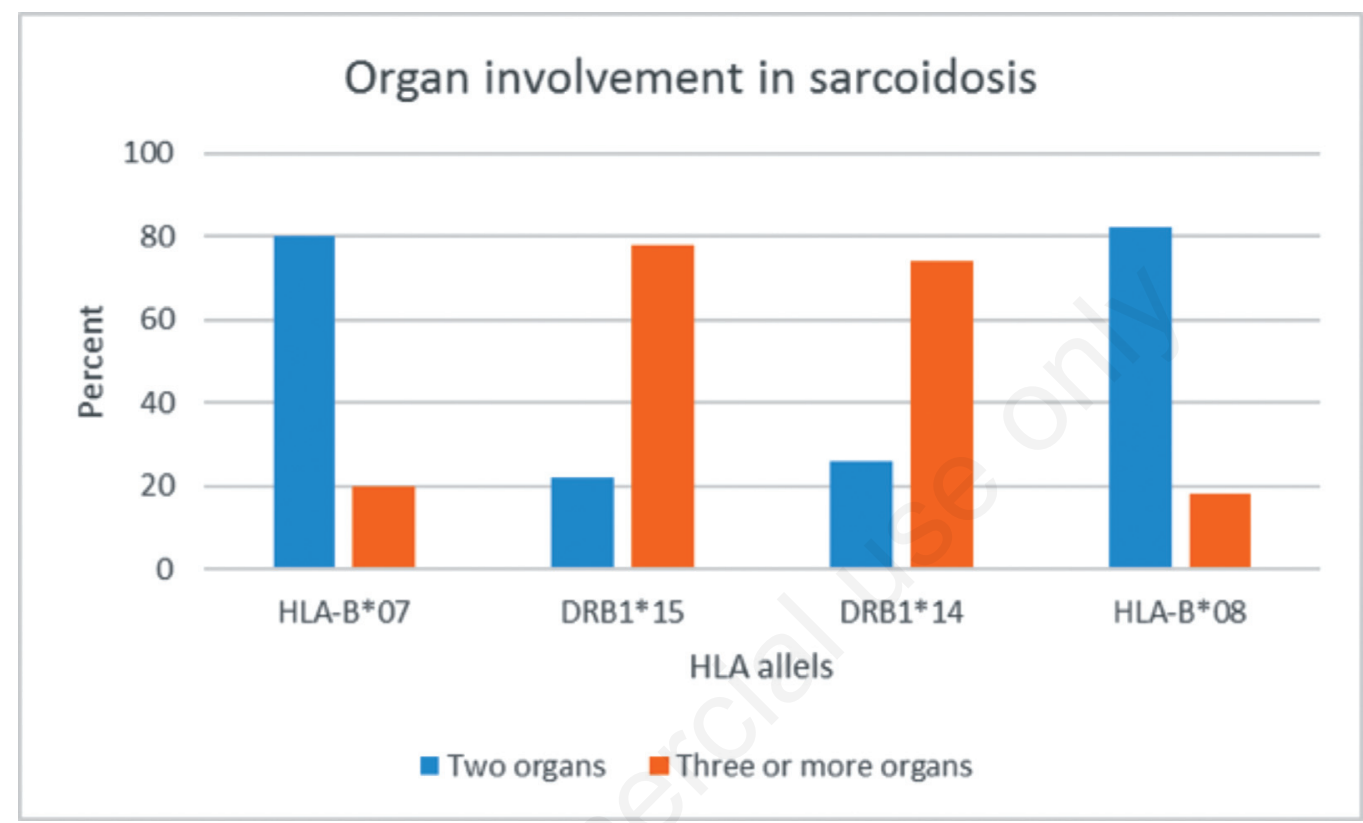

Figure 2. Organ involvement and HLA association.

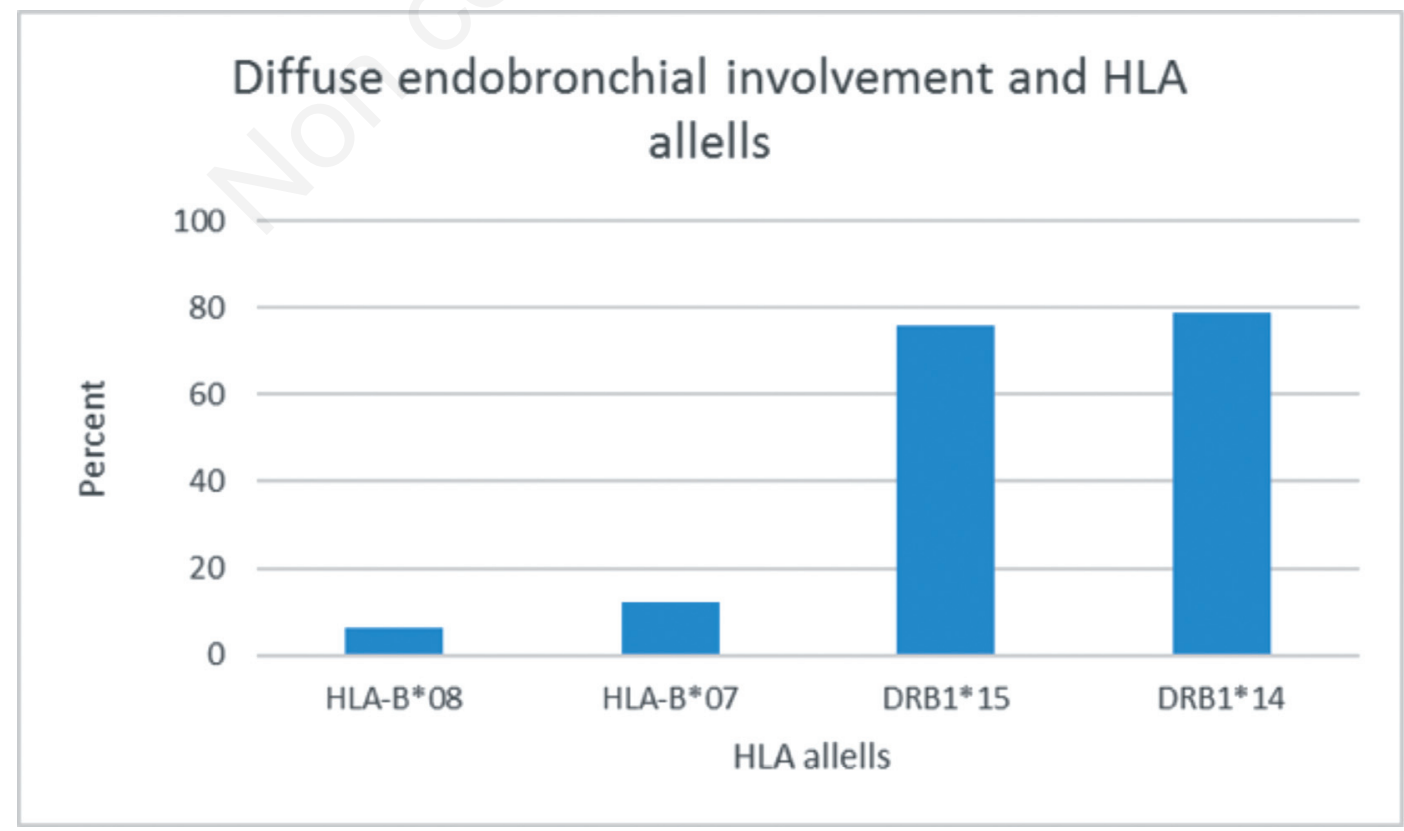

Figure 3. Endobronchial involvement and HLA association. 


\section{Discussion}

Sarcoidosis is usually a benign disease that may show a variable course in a significant number of patients. Heterogeneity of sarcoidosis arises from the interaction of environmental, humoral, and genetic factors. The synergy of different factors leads to a variable clinical profile thereby causing difficulties in diagnostic and therapeutic options. The genetic influence of sarcoidosis is evident by the presence of different clinical manifestations in distinct ethnic groups and familial clustering has focused on the MCHC region.

In our study, patients with positive HLA-B $* 03$, HLA-B*07, and HLAB*08 allels sarcoidosis had a stable benign course while HLA-DRB ${ }^{*} 07$ and ${ }^{*} 15$ positive patients revealed a significant risk for chronic persistent disease. HLA-DRB1*07, HLA-DRB1*14 and HLA-DRB1*15 were more frequent in patients with extensive extrapulmonary organ involvement. Diffuse endobronchial disease was more common in DRB $1 * 14$ and DRB $1 * 15$ positive patients. Our results suggest that the prognostic outcome in sarcoidosis patients is dominantly dependant on the distinct genetic heterogeneity of the disease. Environmental, humoral, and hormonal factors may also play a role in the chronic and persistent disease. It is a well-known fact that the occasional occurrence of sarcoidosis in the first-degree relatives and identical twins suggests that genetic predisposition plays the most important role in the pathogenesis and thereby in the variable clinical manifestations of sarcoidosis $[5,15,16]$. The genetic background of sarcoidosis patients described by the HLA typing in clinical practice appears to be the most crucial factor determining the variable outcome of sarcoidosis for the individual patient is the most relevant finding of this study.

The clinical expression, natural history, and prognosis of sarcoidosis are highly variable. Sarcoidosis often shows an unpredictable disease course. Clinical manifestations also vary with the organs involved [5-7]. In our study, deteriorating FVC and DLCO values were also more frequent in HLA-DRB1*014 and HLA-DRB ${ }^{*} 15$ positive patients. On the other hand, HLA-DRB $1 * 03$, HLA-B*07, and HLA-B*08 positivity was higher in patients with spontaneous resolution. These allels were associated with mild benign disease with a favourable prognosis. Initial chest $x$-ray revealed more advanced disease in HLADRB $1 * 014$ and HLA-DRB1*015 positive patients. Previous studies revealed that HLA-DRB allels played a crucial role for many aspects of sarcoidosis [9,10,17-20]. Prognosis and outcome of sarcoidosis predominantly depends upon the specific HLA profile of the patient. Our study provides a strong evidence for the genetic basis of sarcoidosis in regard to prognosis, and variable disease activity or severity. These results support that the HLA allels are useful to predict the prognostic outcome and to identify patients with severe clinical manifestations of sarcoidosis.

The other crucial aspect of our study was relevant with extrapulmonary organ and diffuse endobronchial disease. HLA-DRB1*014 and HLA-DRB $1 * 015$ positivity was more frequent in patients with extensive organ involvement while the incidence of DEI was also more common in these patients. Tetikkurt and Yanardag have reported that limited and diffuse endobronchial involvement in sarcoidosis are associated with chronic disease [21-23]. The significant correlation of endobronchial disease with certain HLA allels in our study also implies that the HLADRB1 allels may play a critical role for identifying such patients. The most crucial finding of our study is the high frequency of HLA-DRB1*07, HLA-DRB $1{ }^{*} 14$ and HLA-DRB1*15 allels in patients with progressive chronic disease. The strong correlation between these allels and the severe prognosis appears to be a valuable clinical marker for diagnosing chronic sarcoidosis patients with a serious outcome. Primary target of sarcoidosis treatment is the suppressing the inflammatory response and reducing the granuloma burden in the involved organs, mainly the lung. This treatment option depends on the assumption of inhibiting or precluding granulomatous inflammation that leads to a chronic persistent or fibrotic disease. The major current problem in sarcoidosis is to predict the progression to chronic or persistent disease. Consequently, use of HLA alleles may be the most useful objective predictor for such patients. The high incidence of identical HLA typing among our sibling patients also delineates the genetic susceptibility thereby pointing out to the significance of HLA allels in sarcoidosis.

There are several limitations of our study. The major limitation is the small sample size. Studies with larger sample sizes and more heterogeneous patient profiles may be needed to define the potential usefulness of HLA typing for sarcoidosis in regard to extrapulmonary involvement, endobronchial disease, and prognosis. Heterogeneity of sarcoidosis does not only arise from genetic factors. The interaction of environmental, humoral, and even hormonal effects may also play a role in the clinical manifestations and the prognosis. We did not investigate the influence of environmental and humoral circumstances in our study. Sarcoidosis has a higher incidence and a more severe disease course in black people and different racial groups tend to present with various other phenotypes of disease [24-26]. The patient population of our study consisted of only Caucasian patients that may be considered as the third limiting factor since both racial and interracial effects are decisive in sarcoidosis. Further studies including the genetic, epidemiologic, environmental and humoral factors are needed to identify the definitive and precise role of HLA antigens for a variable outcome and chronic disease in sarcoidosis patients.

HLA-DR antigens the hallmark of human immune reaction regulating the host response mechanisms to disorders through the cell-mediated immunity. HLA molecules are pivotal for adaptive immune response and various associations with diseases including sarcoidosis have been reported. These antigens play an extremely important role in the pathogenesis of sarcoidosis and HLA frequencies in sarcoidosis have been investigated by many authors [27-31]. In conclusion, our study points out that the variability of sarcoidosis is strongly associated with the HLA structure of the individual patient. Prognosis, organ involvement, and endobronchial disease in sarcoidosis mainly depend upon the presence of different HLA allels. HLA antigens are useful for detecting patients with diffuse endobronchial disease and extensive extrapulmonary organ involvement. Identification of HLA allels may predict the individual patient profile and the risk for a severe outcome, and thereby determine the patients who require a more meticulous clinical monitoring and treatment in regard to a progressive disease in sarcoidosis.

\section{References}

1. [No authors listed]. Statement on sarcoidosis. Joint Statement of the American Thoracic Society (ATS), the European Respiratory Society (ERS) and the World Association of Sarcoidosis and Other Granulomatous Disorders (WASOG) adopted by the ATS Board of Directors and by the ERS Executive Committee, February. Am J Respir Crit Care Med 1999;160:736-55.

2. Drent M, Strookappe B, Hoitsma E, De Vries J. Consequences of sarcoidosis. Clin Chest Med 2015;36:727-9.

3. Valeyre D, Prasse A, Nunes H, et al. Sarcoidosis. Lancet 2014; 383:1155-67.

4. Wisjenbeek MS, Culver DA. Treatment of Sarcoidosis. Clin Chest Med 2015;36:751-67.

5. Sverrild A, Backer V, Kyvik KO, et al. Heredity in sarcoidosis: a registry-based twin study. Thorax 2008;63:894-6. 
6. Berlin M, Fogdell-Hahn A, Olerup 0, et al. HLA-DR Predicts the prognosis in Scandinavian patients with sarcoidosis. Am J Respir Crit Care Med 1997;156:1601-5.

7. Levin AM, Adrianto I, Datta I, et al. Association of HLA-DRB1 with sarcoidosis susceptibility and prognosis in African Americans. Am J Respir Cell Mol Biol 2015;53:206-16.

8. Ozyilmaz E, Goruroglu Ozturk 0, Yunsel D, et al. Could HLADRB1*11 allel be a clue for extrapulmonary sarcoidosis? Sarcoidosis Vasc Diffuse Lung Dis 2014;31:154-62.

9. Grunewald J, Eklund A. Löfgren's syndrome: human leukocyte antigen strongly influences the disease course. Am J Respir Crit Care Med 2009;179:307-12.

10. Fischer A, Schmid B, Ellinghaus D, et al. A novel sarcoidosis risk locus for Europeans on chromosome 11q13.1. Am J Respir Crit Care Med 2012;1;186:877-85.

11. DeRemee RA. The roentgenographic staging of sarcoidosis: historic and contemporary perspectives. Chest 1983;83:128-32.

12. American Thoracic Society. Lung function testing: selection of reference values and interpretative strategies. Am Rev Respir Dis 1991;144:1202-18.

13. Baughman RP, Costabel U, du Bois RM. Treatment of sarcoidosis. Clin Chest Med 2008;29:533-48.

14. Lazar CA, Culver DA. Treatment of sarcoidosis. Semin Respir Crit Care Med 2010;31:501-18.

15. Nagai S, Shigematsu M, Hamada K, Izumi T. Clinical courses and prognoses of pulmonary sarcoidosis. Curr Opin Pulm Med 1999;5:293-8.

16. Brenna NJ, Crean P, Long JP, Fitzgerald MX. High prevalence of familial sarcoidosis in Irish population. Thorax 1984;39:14-8.

17. Dubrey S, Shah S, Hardman T, Sharma R. Sarcoidosis: the links between epidemiology and aetiology. Postgrad Med J 2014;90:582-9.

18. Grunewald J, Kaiser Y, Ostadkarampour M, et al. T-cell receptorHLA-DRB1 associations suggest specific antigens in pulmonary sarcoidosis. Eur Respir J 2016;47:898-09.

19. Wennerström A, Pietinalho A, Vauhkonen H, et al. HLA-DRB1 allele frequencies and C4 copy number variation in Finnish sarcoidosis patients and associations with disease prognosis. Eur Respir J 2013;42:550-3.

20. Voorter CEM, Drent Voorter, van den Berg-Loonen EM. Severe pulmonary sarcoidosis is strongly associated with the haplotype HLADQB1*0602-DRB1*150101. Hum Immunol 2005;66:826-35.

21. Tetikkurt C, Yanardag H, Bilir M, et al. Clinical features and prognostic significance of limited and diffuse endobronchial sarcoidosis. Br J Med Res 2106;11:1-7.

22. Yanardag H, Tetikkurt C, Bilir M, et al. Clinical features and prognostic significance of endobronchial sarcoidosis. British Br J Med Res 2105;9:1-7.

23. Tetikkurt C. Endobronchial involvement in sarcoidosis. US Resp Pulm Dis 2016;1:27-9.

24. Baughman RP, Terstein AS, Judson MA et al. Clinical characteristics of patients in a case-control study of sarcoidosis. Am J Respir Crit Care Med 2001;164:1885-89.

25. Rybicki BA, Major M, Popovich J, et al. Racial differences in sarcoidosis incidence: a 5-year study in a health maintenance organization. Am J Epidemiol 1997;145:234-41.

26. Gideon MN, Mannino DM. Sarcoidosis mortality in the United States,1979-1991: an analysis of multiple-cause mortality data. Am J Med 1996;100:423-7.

27. Klein J, Sato A. The HLA system. First of two parts. N Engl J Med 2000;343:702-9.

28. Klein J, Sato A. The HLA system. Second of two parts. N Engl J Med 2000;343:782-6.

29. Abe S, Yamaguchi E, Makimura S, et al. Association of HLA-DR with sarcoidosis: correlation with clinical course. Chest 1987;92: 488-90.

30. Sato H, Grutters JC, Pantelidis P, et al. HLA-DQB1*0201: a marker for good prognosis in British and Dutch patients with sarcoidosis. Am J Respir Cell Mol Biol 2002;27:406-12.

31. Schurmann M, Lympany PA, Reichel P, et al. Familial sarcoidosis linked to the major histocompability complex antigen. Am J Respir Crit Care Med 2000;162:861-4. 